\title{
Tracking Control of Mobile Manipulators Based on Decentralized Control
}

\author{
Raouf Fareh $^{1}$, Sofiane Khadraoui ${ }^{1}$, Maarouf Saad ${ }^{2}$, and Maamar Bettayeb ${ }^{1}$ \\ ${ }^{1}$ Department of Electrical and Computer Eng., University of Sharjah, P.O.Box 27272, Sharjah, UAE \\ rfareh@sharjah.ac.ae; skhadraoui@sharjah.ac.ae; maamar@sharjah.ac.ae \\ ${ }^{2}$ Electrical Engineering Department, Université du Québec, École de technologie supérieure, 1100, rue Notre-Dame ouest, \\ Montréal (Québec), H3C 1K3, Canada \\ maarouf.saad@etsmtl.ca
}

\begin{abstract}
This paper presents a decentralized control strategy for mobile manipulator systems. First, the whole system of mobile manipulator is divided into two interconnected subsystems: nonholonomic mobile platform subsystem and holonomic manipulator subsystem. Second, a kinematic and dynamic controllers are developed for mobile platform subsystem. The kinematic control provides the desired velocity that will be used to develop the dynamic controller that ensures the tracking of the desired trajectories of the mobile platform subsystem. Third, the control law of the manipulator subsystem is developed based on the desired and real values of the manipulator subsystem as well as the desired velocity of the platform subsystem. Lyapunov approach is used to prove the stability of the tracking error of both subsystems. Simulation results on 2 DOF manipulator mounted on a mobile platform are given to demonstrate the feasibility and effectiveness of the proposed approach.
\end{abstract}

Keywords: Mobile manipulator, trajectory tracking, decentralized control, stability.

\section{Introduction}

The tracking control still a challenging problem for the mobile manipulator due to the complexity and strong coupled dynamics of the mobile platform and the robotic arm. Many control strategies have been developed in recent years to track a desired trajectories of mobile manipulators. Two categories of the control schemes are usually used for this kind of systems. First, the mobile manipulator is viewed as one multivariable system where one control law is used for the whole system. In this case, many control strategies have been applied to mobile manipulators. The position control problem of mobile manipulators operating in the task space with state constraints was proposed in [1]. A visual dynamic control based on passivity is developed in [2] to solve the target tracking problem of mobile manipulators. A planning and control algorithm for coordinating the motion of a mobile manipulator is presented in [3]. The authors presented a control algorithm so that the manipulator was always positioned at preferred configuration measured by its manipulability. In order to simplify the nonlinear model and to design a controller, output feedback linearization was used. Model-based PD-like controller was also used in [4] to eliminate the tracking errors of the mobile manipulator. PD and neural network controllers for the overall mobile manipulator were proposed in [5].

All these techniques use the mobile manipulator as one multivariable system and the overall dynamic system contains $(p+m)$ degrees of freedom (DOF) where $p$ and $m$ are the number of DOF of the mobile platform and the manipulator, respectively. This is may after all be acceptable as a good choice when $(p+m)$ is small. However, the complexity of the computations increases geometrically with $(p+m)$ [6] . To overcome this problem, a second category of the control scheme which consists of dividing the dynamics of the mobile manipulator into two interconnected subsystems: mobile platform and manipulator. Many control strategies have been proposed for the mobile manipulators using this configuration. Decentralized robust controllers for mobile manipulators were proposed in [7] to track a desired trajectory in the workspace. The dynamical models of the two subsystems included the reaction forces. However, the angular motion was excluded and only translation of the mobile platform was considered. In [8], two interaction controllers were developed. The first control law consists of a robust adaptive controller for the manipulator subsystem while the second one is an input-output linearizing controller for the mobile platform subsystem. The control strategy was employed to 
minimize the adverse effect of wheel slip on track performances. A kinematic controller of a mobile manipulator with uncertainties was proposed in [9]. The dynamic surface control technique was applied in [10] for mobile manipulators.

This paper takes the advantage of the decentralized configuration which uses the mobile manipulator as two interconnected subsystems: the mobile platform subsystem and the manipulator subsystem. The considered system is a two-link manipulator mounted on a mobile platform. Since this desired velocity cannot be generated directly by the motors, a control torques are designed for the mobile platform based on the dynamic model. Next, a control law for the manipulator subsystem is developed based on Lyapunov approach. Simulation results show effectiveness of the proposed decentralized control strategy for controlling the mobile manipulator.

The rest of this paper is organized as follows. In Section 2, system description and modelling of mobile manipulators are addressed. The control law for the mobile platform subsystem is presented in section 3. Section 4 presents the control law and the stability of the manipulator subsystem. The simulation results are given in Section 5, and the conclusion is presented in Section 6.

\section{System Description and Modelling}

The mobile manipulator considered is composed of a wheeled mobile platform and a two-link manipulator as shown in Fig.1. The mobile platform moves by driving two independent wheels.

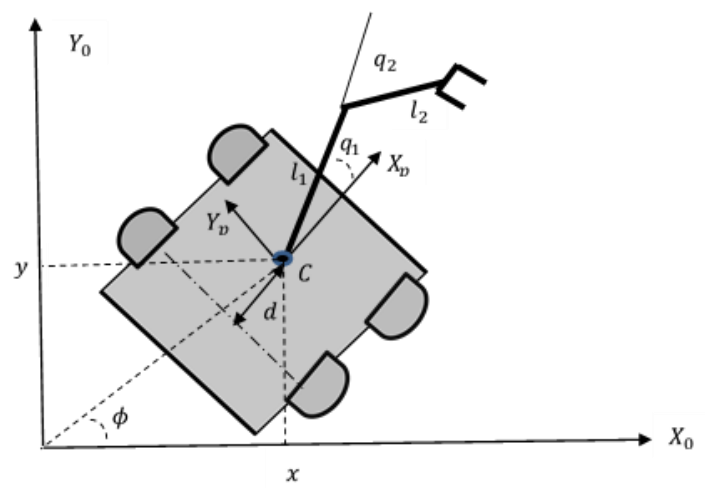

Fig. 1. 2DOF Mobile manipulator.

In addition, the manipulator is generally considered to be a holonomic system, while the mobile platform is subject to nonholonomic constraint and hence, the mathematical model of mobile manipulator can be expressed as [11]:

$$
M(q) \ddot{q}+C(q, \dot{q}) \dot{q}+G(q)=B(q) \tau-A^{T}(q) \lambda
$$

The nonholonomic is given as follows:

$$
A(q) \dot{q}=0
$$

where $M(q) \in \mathcal{R}^{n \times n}$ is an inertia and mass matrix, $C(q, \dot{q}) \dot{q}$ is the Coriolis vector, $G(q) \in \mathcal{R}^{n}$ is a vector of gravity terms. $q \in \mathcal{R}^{n}$ denotes the vector of the generalized positions in the joint space, $\dot{q}$ and $\ddot{q}$ are the velocity and acceleration vectors, respectively. Let $q=\left[\begin{array}{ll}q_{v}^{T} & q_{r}^{T}\end{array}\right]^{T}$, where $q_{v} \in \mathcal{R}^{p}$ represents the position and orientation of the mobile platform subsystem, $q_{r} \in \mathcal{R}^{m}$ represents the link position of the manipulator subsystem, and $n=p+m . A(q) \in \mathcal{R}^{r \times n}$ is the constraint matrix, $\lambda \in \mathcal{R}^{r}$ is the constraint force. $B(q) \in \mathcal{R}^{p \times(p-r)}$ is the input transformation matrix. $\tau \in \mathcal{R}^{n-r}$ is the input torque.

Since the nonholonomic characteristics of the mobile manipulator is caused by the movement of the mobile platform, Equation (2) can be simplified to:

$$
A_{v}\left(q_{v}\right) \dot{q}_{v}=0
$$

where $A_{v}\left(q_{v}\right) \in \mathcal{R}^{r \times p}$ is the constraint matrix of mobile platform. 
The dynamic model of the mobile manipulator given in (1) can take the following form:

$$
\left[\begin{array}{ll}
M_{11} & M_{12} \\
M_{21} & M_{22}
\end{array}\right]\left[\begin{array}{l}
\ddot{q}_{v} \\
\ddot{q}_{r}
\end{array}\right]+\left[\begin{array}{ll}
C_{11} & C_{12} \\
C_{21} & C_{22}
\end{array}\right]\left[\begin{array}{l}
\dot{q}_{v} \\
\dot{q}_{r}
\end{array}\right]+\left[\begin{array}{l}
G_{1} \\
G_{2}
\end{array}\right]=\left[\begin{array}{c}
B_{v}\left(q_{v}\right) \tau_{v} \\
\tau_{r}
\end{array}\right]-\left[\begin{array}{c}
A_{v}^{T}\left(q_{v}\right) \lambda \\
0
\end{array}\right]
$$

where $\tau_{v} \in \mathcal{R}^{p-r}$ is the control torque of mobile platform, $\tau_{r} \in \mathcal{R}^{m}$ is the control torque of manipulator, $M_{11}$ and $M_{22}$ represent the inertia matrices of mobile platform and manipulator respectively, $M_{12} \ddot{q}_{r}$ and $M_{21} \ddot{q}_{v}$ represent the interaction inertia between the manipulator and mobile platform, $C_{12} \dot{q}_{r}$ and $C_{21} \dot{q}_{v}$ also represent the interaction centripetal and Coriolis forces between two subsystems. The model has the following properties that will be used in the stability analysis of the developed control law:

P1. The inertia-mass matrix $M(q)$ is symmetric positive definite.

P2: The inertia-mass matrix $M(q)$ and the Coriolis matrix $C(q, \dot{q})$ satisfy the following skew-symmetric property:

$$
X^{T}(\dot{M}(q, \dot{q})-2 C(q, \dot{q})) X=0 \quad \forall X \in \mathcal{R}^{n}
$$

\section{Control of Mobile Platform Subsystem}

In this section, the control torque based on dynamic model is developed for mobile platform subsystem. First, the velocity control based on kinematic model is designed to develop the desired velocity. Next, the torques for mobile platform are developed using this desired velocity.

The dynamic equation of mobile platform subsystem is derived from (4) as follows:

$$
M_{11} \ddot{q}_{v}+M_{12} \ddot{q}_{r}+C_{11} \dot{q}_{v}+C_{12} \dot{q}_{r}+G_{1}=B_{v}\left(q_{v}\right) \tau_{v}-A_{v}^{T}\left(q_{v}\right) \lambda
$$

As mentioned $A_{v}$ is the constrain matrix. When selecting a full rank matrix $S\left(q_{v}\right) \in \mathcal{R}^{p \times(p-r)}$ to be a basis of null space $A_{v}\left(q_{v}\right)$, the constraint equation will be:

$$
S^{T}\left(q_{v}\right) A_{v}^{T}\left(q_{v}\right)=0
$$

There exists an auxiliary input vector $\in \mathcal{R}^{p-r}$, and satisfying

$$
\dot{q}_{v}=S\left(q_{v}\right) V(t)
$$

where $\dot{q}_{v}=\left[\begin{array}{lll}\dot{x} & \dot{y} & \dot{\varnothing}\end{array}\right]^{T}$ and $V=\left[\begin{array}{ll}v & w\end{array}\right]^{T}, v$ and $w$ are the linear and angular velocity.

When multiplying Eq. (6) by $S^{T}$, the constraint force term $A_{v}^{T}\left(q_{v}\right) \lambda$ can be eliminated. So, we have:

$$
S^{T} M_{11} \ddot{q}_{v}+S^{T} M_{12} \ddot{q}_{r}+S^{T} C_{11} \dot{q}_{v}+S^{T} C_{12} \dot{q}_{r}+S^{T} G_{1}=S^{T} B_{v} \tau_{v}
$$

Introducing (8), the dynamic equation (9) becomes:

$$
\bar{M}_{11} \dot{V}+\bar{C}_{11} V+f_{1}=\bar{\tau}_{v}
$$

where $\bar{M}_{11}=S^{T} M_{11} S ; \bar{C}_{11}=S^{T} M_{11} \dot{S}+S^{T} C_{11} S$ and $\bar{\tau}_{v}=S^{T} B_{v} \tau_{v}$

The dynamic coupling term that is caused by the manipulator and the gravity are given as follows:

$$
f_{1}=S^{T}\left(M_{12} \ddot{q}_{r}+C_{12} \dot{q}_{r}+G_{1}\right)
$$

From the modified model, we present the following properties that will be used in the stability analysis:

P3. The inertia-mass matrices $\bar{M}_{11}$ and the Coriolis matrices $\bar{C}_{11}$ satisfy the following skew-symmetric property: 


$$
X^{T}\left(\dot{\bar{M}}_{11}-2 \bar{C}_{11}\right) X=0 \quad \forall X \in \mathcal{R}^{p}
$$

The kinematic model of two-wheel driven mobile platform can be expressed as [7]:

$$
\dot{q}_{v}=\left[\begin{array}{c}
\dot{x} \\
\dot{y} \\
\dot{\phi}
\end{array}\right]=\left[\begin{array}{cc}
\cos \phi & -d \sin \phi \\
\sin \phi & d \cos \phi \\
0 & 1
\end{array}\right]\left[\begin{array}{l}
v \\
\omega
\end{array}\right]
$$

where $(x, y)$ is the coordinate of point $\mathrm{C}$ in the coordinate system XOY (Figure 1 ); $\emptyset$ is the direction angle when the mobile platform rotates around the $\mathrm{X}$-axis anticlockwise; $\mathrm{d}$ is the distance between the point $\mathrm{C}$ of mobile platform and the axis mid-points $\mathrm{G}$ of two driven wheels. $v$ and $\omega$ represent the linear and angular velocities of mobile platform, respectively.

Note that the objective is to track a reference trajectory by the mobile platform. Then, the desired position is $q_{v d}=$ $\left[\begin{array}{lll}x_{d} & y_{d} & \phi_{d}\end{array}\right]$ and the desired velocity is $V_{d}=\left[\begin{array}{ll}v_{d} & \omega_{d}\end{array}\right]^{T}$. Therefore, the tracking errors is obtained using the Kanayama transformation [12] as follows:

$$
\left[\begin{array}{c}
\tilde{x} \\
\tilde{y} \\
\tilde{\phi}
\end{array}\right]=\left[\begin{array}{ccc}
\cos \phi & \sin \phi & 0 \\
-\sin \phi & \cos \phi & 0 \\
0 & 0 & 1
\end{array}\right]\left[\begin{array}{l}
x_{d}-x \\
y_{d}-y \\
\phi_{d}-\phi
\end{array}\right]
$$

Proposition 1: The error dynamics (14) are asymptotically stable when using the following velocity control law:

$$
V(t)=\left[\begin{array}{c}
v \\
\omega
\end{array}\right]=\left[\begin{array}{c}
k_{x} \tilde{x}+v_{d} \cos \tilde{\phi} \\
\omega_{d}+k_{y} v_{d} \tilde{y}+k_{\phi} v_{d} \sin \tilde{\phi}
\end{array}\right]
$$

where $k_{x}, k_{y}$ and $k_{\phi}$; are positive controller gains.

To prove proposition 2, we consider the following positive Lyapunov function:

$$
W=\frac{1}{2} \tilde{x}^{2}+\frac{1}{2} \tilde{y}^{2}+\frac{1-\cos \tilde{\phi}}{k_{y}}
$$

It has been proven that the kinematic system of mobile robot, which consisted of Eq. (13), and (15), is closed loop stable [7].

The above velocity control law Eq. (15) is only designed specially based on the kinematic model. However, the motors generate control torque and cannot generate directly the velocity control. Therefore, it is necessary to design the torques for mobile platform based on the dynamic model and then the control torque will result in an actual velocity. Using the actual and desired velocities, the velocity tracking errors can be expressed as:

$$
z=V-V_{d}
$$

where $V$ and $V_{d}$ are the actual and desired velocity of mobile platform, respectively. From the dynamic model (10), the term $f_{1}$ existing in (11) contains the acceleration $\ddot{q}_{r}$. In order to avoid this acceleration we define the sliding surface of the manipulator subsystem as follows:

$$
r=\dot{e}+K e
$$

where $e=q_{r d}-q_{r}$ is the position error. Thus, the generalized coordinate $\dot{q}_{r}$ and $\ddot{q}_{r}$ can be written as follows: 


$$
\dot{q}_{r}=\dot{q}_{r d}-\dot{e}=\dot{q}_{r d}-r+K e ; \ddot{q}_{r}=\ddot{q}_{r d}-\ddot{e}=\ddot{q}_{r d}-\dot{r}+K \dot{e}
$$

Using equation (17), the dynamic equation (10) is equivalent to the following equation:

$$
\bar{M}_{11} \dot{z}+\bar{C}_{11} Z+\bar{M}_{11} \dot{V}_{d}+\bar{C}_{11} V_{d}+f_{1}=\bar{\tau}_{v}
$$

Proposition 2: The resulting error dynamics is asymptotically stable when using the following control law:

$$
\bar{\tau}_{v}=\bar{M}_{11} \dot{V}_{d}+\bar{C}_{11} V_{d}+f_{1}-K_{d v} Z
$$

where $K_{d v}$ is positive gain

To prove the stability, let find the error dynamics by inserting the platform controller (21) in the dynamic equation (20). So, the resulting error dynamics can be expressed as follows:

$$
\bar{M}_{11} \dot{z}+\bar{C}_{11} z+K_{d v} z=0
$$

To prove the stability of the error dynamics, we consider the following positive Lyapunov function:

$$
V_{1}=\frac{1}{2} z^{T} \bar{M}_{11} z
$$

The time derivative is : $\dot{V}_{1}=z^{T} \bar{M}_{11} \dot{Z}+\frac{1}{2} z^{T} \dot{\bar{M}}_{11} z$

Using the error dynamics (22), we get: $\dot{V}_{1}=z^{T}\left[-\bar{C}_{11} z-K_{d v} z\right]+\frac{1}{2} z^{T} \dot{\bar{M}}_{11} z$

Using the property (12), $\dot{V}_{1}$ becomes:

$$
\dot{V}_{1}=-z^{T} K_{d v} Z
$$

Since $K_{d v}$ is positive definite matrix, the time derivative $\dot{V}_{1}$ is negative. Using LaSalle theorem [13], the error dynamics are asymptotically stable.

\section{Control of Manipulator Subsystem}

This section presents the control strategy for the manipulator subsystem. From Eq. (4), the dynamic equation of manipulator subsystem is expressed as:

$$
M_{21} \ddot{q}_{v}+M_{22} \ddot{q}_{r}+C_{21} \dot{q}_{v}+C_{22} \dot{q}_{r}+G_{2}=\tau_{r}
$$

This dynamic equation contains the acceleration term $\ddot{q}_{r}$. To avoid this problem, the equation (19) can be used. The dynamic equation can be rearranged as follows:

$$
\begin{aligned}
& M_{21} \ddot{q}_{v}+M_{22}\left(\ddot{q}_{r d}-\dot{r}+K \dot{e}\right)+C_{21} \dot{q}_{v}+C_{22}\left(\dot{q}_{r d}-r+K e\right)+G_{2}=\tau_{r} \\
& -M_{22} \dot{r}+M_{21} \ddot{q}_{v}+M_{22} K \dot{e}+C_{21} \dot{q}_{v}-C_{22}(r-K e)+C_{22} \dot{q}_{r d}+M_{22} \ddot{q}_{r d}+G_{2}=\tau_{r} \\
& -M_{22} \dot{r}+M_{21} \ddot{q}_{v}+M_{22} K(r-K e)+C_{21} \dot{q}_{v}-C_{22}(r-K e)+C_{22} \dot{q}_{r d}+M_{22} \ddot{q}_{r d}+G_{2}=\tau_{r} \\
& -M_{22} \dot{r}+M_{21} \ddot{q}_{v}+C_{21} \dot{q}_{v}+\left(M_{22} K-C_{22}\right)(r-K e)+C_{22} \dot{q}_{r d}+M_{22} \ddot{q}_{r d}+G_{2}=\tau_{r}
\end{aligned}
$$

Thus, the equation (25) is equivalent to the following expression:

$$
-M_{22} \dot{r}+\left(M_{22} K-C_{22}\right)(r-K e)+f_{2}=\tau_{r}
$$

where 


$$
f_{2}=M_{21} \ddot{q}_{v}+C_{21} \dot{q}_{v}+C_{22} \dot{q}_{r d}+M_{22} \ddot{q}_{r d}+G_{2}
$$

Using the equations (8), $f_{2}$ can be written as:

$$
\begin{aligned}
f_{2} & =M_{21}(S \dot{V}+\dot{S} V)+C_{21} S V+C_{22} \dot{q}_{r d}+M_{22} \ddot{q}_{r d}+G_{2} \\
& =M_{21} S \dot{V}+\left(M_{21} \dot{S}+C_{21} S\right) V+C_{22} \dot{q}_{r d}+M_{22} \ddot{q}_{r d}+G_{2}
\end{aligned}
$$

Let propose now the following control law for the manipulator subsystem:

$$
\tau_{r}=M_{22} K r-\left(C_{22}-M_{22} K\right) K e+f_{2}+K_{d r} r
$$

The error dynamics can be determined by inserting the control law (28) in the dynamic equation of (26) as follows:

$$
-M_{22} \dot{r}+\left(M_{22} K-C_{22}\right)(r-K e)+f_{2}=M_{22} K r-\left(C_{22}-M_{22} K\right) K e+f_{2}+K_{d r} r
$$

After simplification, the error dynamics can be expressed as:

$$
M_{22} \dot{r}+C_{22} r+K_{d r} r=0
$$

Proposition 3: using the control law (28), the error dynamics (30) of the manipulator subsystem are asymptotically stable when using the following positive Lyapunov function:

$$
V_{2}=\frac{1}{2} r^{T} M_{22} r
$$

Proof: The time derivative of $V_{2}$ is $: \dot{V}_{2}=r^{T} M_{22} \dot{r}+\frac{1}{2} r^{T} \dot{M}_{22} r$

Using the error dynamics (30), we get: $\dot{V}_{2}=r^{T}\left[-C_{22} r-K_{d r} r\right]+\frac{1}{2} r^{T} \dot{M}_{22} r$

Using the property (5), $\dot{V}_{2}$ becomes:

$$
\dot{V}_{2}=-r^{T} K_{d r} r
$$

Since $K_{d r}$ is positive definite matrix, the time derivation $\dot{V}_{2}$ is negative. Using LaSalle theorem [13], the error dynamics is asymptotically stable.

\section{Simulation results}

The mobile manipulator which is shown in Fig. 1 is utilized to demonstrate the effectiveness of the proposed decentralized control strategy. The nonholonomic constraint is given as

$$
\dot{y}_{c} \cos \emptyset-\dot{x}_{c} \sin \emptyset-d \dot{\varnothing}=0
$$

Then, the constraint matrix of nonholonomic system corresponding to Eq. (3) can be obtained as

$$
A_{v}\left(q_{v}\right)=\left[\begin{array}{lll}
-\sin \emptyset & \cos \emptyset & -d
\end{array}\right]
$$

The matrix $S\left(q_{v}\right)$ as a basis in null space $A_{v}\left(q_{v}\right)$ and the corresponding parameters of the dynamic model of mobile manipulator, which is shown in Eq. (4), are given as: 
$S\left(q_{v}\right)=\left[\begin{array}{cc}\frac{r}{2} \cos \emptyset+\frac{r}{R} d \sin \emptyset & \frac{r}{2} \cos \emptyset-\frac{r}{R} d \sin \emptyset \\ \frac{r}{2} \sin \emptyset-\frac{r}{R} d \cos \emptyset & \frac{r}{2} \sin \emptyset+\frac{r}{R} d \cos \emptyset \\ -\frac{r}{R} & \frac{r}{R}\end{array}\right] ; B_{11}=\frac{1}{r}\left[\begin{array}{cc}\cos \emptyset & \cos \emptyset \\ \sin \emptyset & \sin \emptyset \\ -R & R\end{array}\right]$

$M_{11}=\left[\begin{array}{ccc}m_{o 12}+\frac{2 l_{w} \sin ^{2} \emptyset}{r^{2}} & -\frac{2 l_{w} \sin \phi \cos \emptyset}{r^{2}} & -2 m_{12} d \sin \emptyset \\ -\frac{2 l_{w} \sin \phi \cos \emptyset}{r^{2}} & m_{o 12}+\frac{2 l_{w} \cos ^{2} \emptyset}{r^{2}} & 2 m_{12} d \cos \emptyset \\ -2 m_{12} d \sin \emptyset & 2 m_{12} d \cos \emptyset & m_{33}\end{array}\right] ; C_{11}=\left[\begin{array}{ccc}\frac{2 l_{w} \dot{\phi} \sin \phi \cos \emptyset}{r^{2}} & \frac{l_{w} \dot{\phi} \cos 2 \emptyset}{r^{2}} & -m_{12} d \dot{\phi} \cos \emptyset \\ \frac{l_{w} \dot{\phi} \cos 2 \emptyset}{r^{2}} & -\frac{2 l_{w} \dot{\phi} \sin \phi \cos \emptyset}{r^{2}} & -m_{12} d \dot{\emptyset} \sin \emptyset \\ -m_{12} d \dot{\phi} \cos \emptyset & -m_{12} d \dot{\phi} \sin \emptyset & 0\end{array}\right]$

$M_{12}=M_{21}^{T}=\left[\begin{array}{ccc}0 & 0 & I_{12} \\ 0 & 0 & 0\end{array}\right] ; M_{22}=\left[\begin{array}{cc}I_{12} & 0 \\ 0 & I_{2}\end{array}\right] ; F_{1}=\left[\begin{array}{l}0 \\ 0 \\ 0\end{array}\right] ; ; C_{12}=C_{21}^{T}=\left[\begin{array}{ccc}0 & 0 & 0 \\ 0 & 0 & 0\end{array}\right] ; C_{22}=\left[\begin{array}{cc}0 & 0 \\ 0 & 0\end{array}\right] ; F_{1}=\left[\begin{array}{c}0 \\ m_{2} g l_{2} \\ \sin \theta_{2}\end{array}\right] ;$

Where $m_{o 12}=m_{0}+m_{1}+m_{2} ; \quad m_{12}=m_{1}+m_{2} ; \quad$ mat $_{33}=I_{o 12}+m_{12} d^{2}+2 I_{w} R^{2} / r^{2} ; \quad I_{o 12}=I_{0}+I_{1}+I_{2} ;$ $I_{12}=I_{1}+I_{2} m_{0}$ is the mass of mobile platform, $\mathrm{r}$ is drive wheel radius of mobile platform; $2 \mathrm{R}$ is the distance between two drive wheels; $I_{w}$ is the moment inertia of platform which rotates around central axis, $I_{0}$ is the moment inertia. m1 and $\mathrm{m} 2$ represent the mass of link1 and link2 of manipulator respectively, I1 and $\mathrm{I} 2$ are the moment inertia of link1 and link 2 of manipulator respectively. The mobile manipulator parameters used for the simulation are shown in Table1. For the mobile platform subsystem, consider the kinematic controller, the reference trajectory is chosen as $q_{v d}=\left[\begin{array}{lll}x_{r} & y_{r} & \emptyset_{r}\end{array}\right]^{T}$ then $\dot{x}_{r}=V_{r} \cos \emptyset_{r} ; \dot{y}_{r}=V_{r} \sin \emptyset_{r}, \dot{\emptyset}_{r}=\omega_{r}$. where the linear velocity is chosen as $V_{r}=0.5 m / s$ and the reference angular velocity is chosen as $\omega_{r}=0 \mathrm{rad} / \mathrm{s}$. The reference initial position of mobile platform is $q_{v d}(0)=\left[\begin{array}{lll}2 & 2 & 45^{\circ}\end{array}\right]^{T}$, while the actual initial position is $q_{v}(0)=\left[\begin{array}{lll}1 & 1 & 0^{o}\end{array}\right]^{T}$. The kinematic controller is shown as Eq. (15), where the gains are set to be $k_{x}=5 ; k_{y}=8$; and $k_{\phi}=10$. The dynamic controller gains for the platform subsystem are $k_{d v}=\operatorname{diag}[20,20]$. For the manipulator subsystem, the controller's gains are $k_{d r}=\operatorname{diag}[15,15]$.

Table 1: Simulation parameters.

\begin{tabular}{|l|l|}
\hline Parameters & values \\
\hline$m_{0}(\mathrm{~kg})$ & 40 \\
\hline$m_{1}(\mathrm{~kg})$ & 6 \\
\hline$m_{2}(\mathrm{~kg})$ & 4 \\
\hline$l_{1}(\mathrm{~m})$ & 1.1 \\
\hline$l_{2}(\mathrm{~m})$ & 0.8 \\
\hline$r(\mathrm{~m})$ & 0.1 \\
\hline $2 \mathrm{R}(\mathrm{m})$ & 0.3 \\
\hline $\mathrm{d}(\mathrm{m})$ & 0.3 \\
\hline$I_{W}\left(\mathrm{kgm}^{2}\right)$ & 2 \\
\hline$I_{0}\left(\mathrm{kgm}^{2}\right)$ & 2 \\
\hline$I_{1}\left(\mathrm{kgm}^{2}\right)$ & 1 \\
\hline$I_{2}\left(\mathrm{kgm}^{2}\right)$ & 1 \\
\hline
\end{tabular}

The simulation parameters are given in Table 1. The simulation results are shown in figure 2, 3 and 4 . 

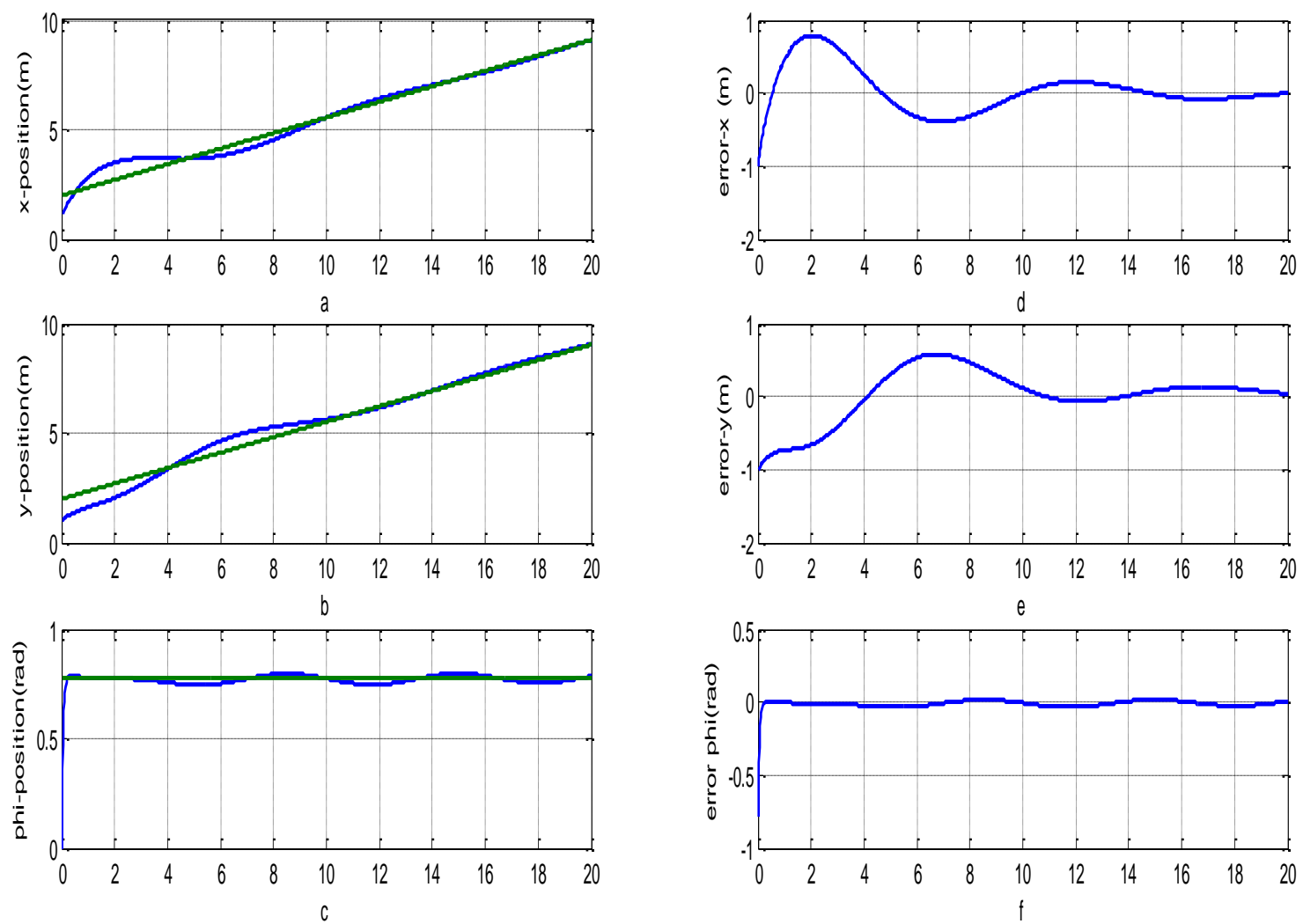

Fig. 2. Mobile platform subsystem. (a) Tracking trajectory of x-position, (b) Tracking trajectory of y-position (c) Tracking trajectory of $\phi$-direction, (d) Tracking error of x-position, (e) Tracking error of y-position (f) Tracking error of $\phi$-direction.

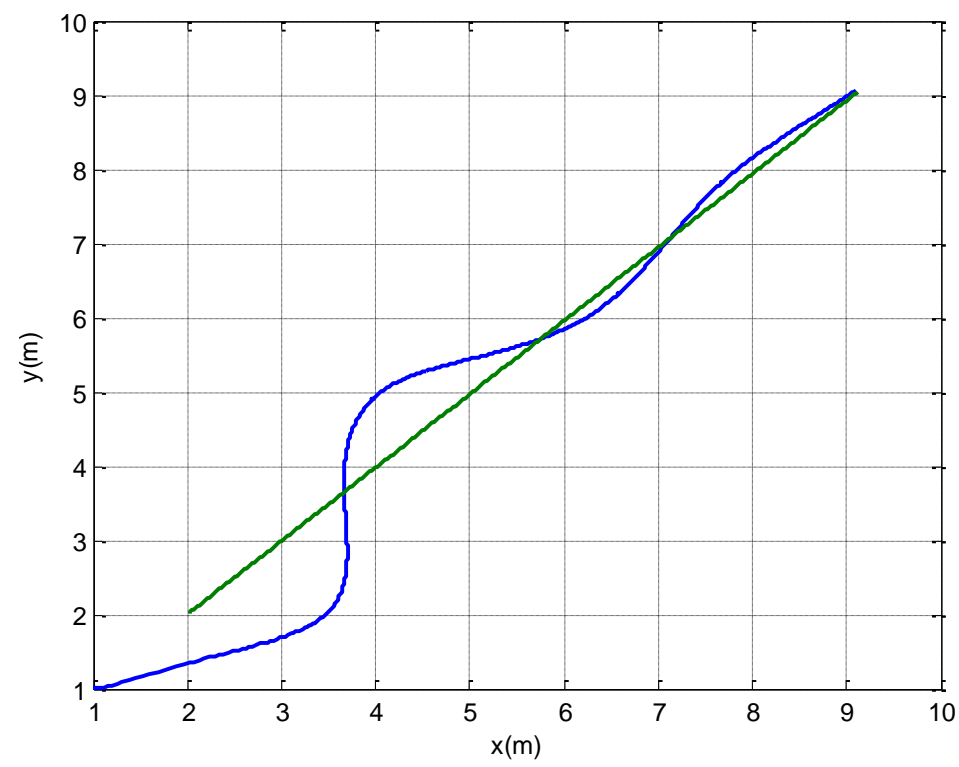

Fig. 3. Tracking trajectory of the platform in $(\mathrm{X}, \mathrm{Y})$ plan. 

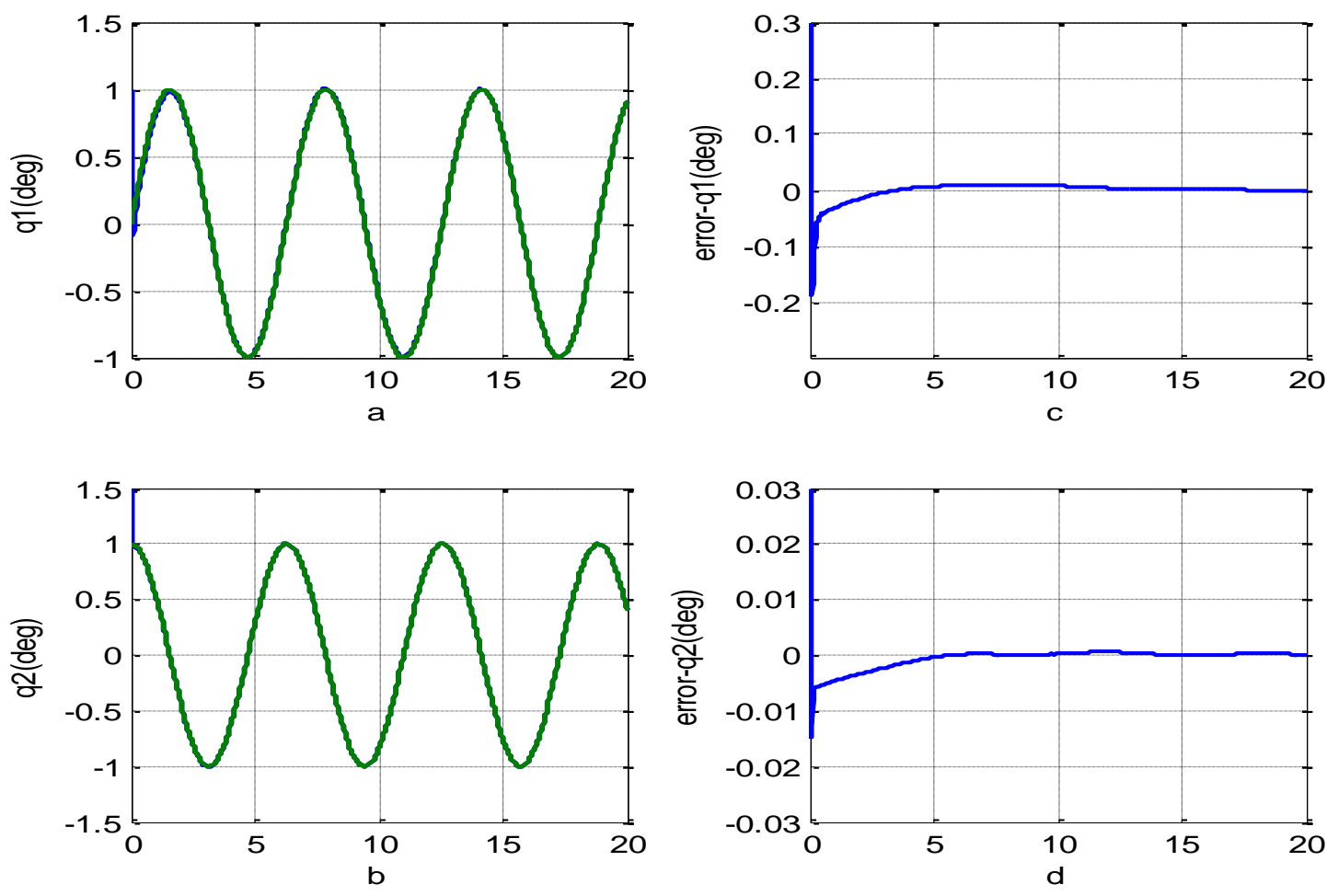

Fig. 4. Manipulator subsystem. (a) Tracking trajectory of link 1 (b) Tracking trajectory of link 2 (c) Tracking error of link 1, (d) Tracking error of link 2 .

For the mobile platform subsystem, a good tracking is shown in figure 2. The desired and real values of $\mathrm{x}, \mathrm{y}$ and $\emptyset$ are presented in figure 2 (a-b-c). This good tracking is conformed by figure 2 (d-e-f) which show the associated tracking errors. Figure 3 shows a good tracking on $\mathrm{x}-\mathrm{y}$ plane of mobile platform. Despite the different initial conditions, the tracking errors converge to zero. For the manipulator subsystem, the tracking trajectories of the first and second link are shown in Figure 4 (a-b) and the corresponding tracking errors are given in Figure 4 (c-d). These figures show a good tracking of the desired trajectories in the joint space of the manipulator.

Finally, from these simulation results, despite the different starting point of the desired and the real values, the steady state errors are very small and converge to zero for both subsystems which show an effective control performance on mobile manipulators.

\section{Conclusion}

This paper presents a decentralized controller for mobile manipulator where it is divided into non-holonomic mobile platform subsystem and holonomic manipulators subsystem. By considering the kinematic controller of mobile platform, the dynamic controller is developed to ensure a good tracking of $\mathrm{x}, \mathrm{y}$ and $\emptyset$. The control law of the manipulator subsystem is developed based on Lyapunov theory. The proposed control method demonstrated an effective control performance on mobile manipulator. As a future work, the proposed decentralized control strategy will be validated experimentally and will be also applied to other kinds of electro- mechanical systems.

\section{References}

[1] M. Galicki, "An adaptive non-linear constraint control of mobile manipulators," Mechanism and Machine Theory, vol. 88, pp. 63-85, 2015.

[2] V. H. Andaluz, F. Roberti, L. Salinas, J. M. Toibero, and R. Carelli, "Passivity-based visual feedback control with dynamic compensation of mobile manipulators: Stability and L2-gain performance analysis," Robotics and 
Autonomous Systems, vol. 66, pp. 64-74, 2015.

[3] Y. Yamamoto and X. Yun, "Coordinating locomotion and manipulation of a mobile manipulator," in Decision and Control, 1992., Proceedings of the 31st IEEE Conference on, 1992, pp. 2643-2648.

[4] E. Papadopoulos and J. Poulakakis, "Planning and model-based control for mobile manipulators," in Intelligent Robots and Systems, 2000.(IROS 2000). Proceedings. 2000 IEEE/RSJ International Conference on, 2000, pp. 18101815.

[5] C.-Y. Lee, I.-K. Jeong, I.-H. Lee, and J.-J. Lee, "Motion control of mobile manipulator based on neural networks and error compensation," in Robotics and Automation, 2004. Proceedings. ICRA'04. 2004 IEEE International Conference on, 2004, pp. 4627-4632.

[6] K. Liu and F. L. Lewis, "Decentralized continuous robust controller for mobile robots," in 1990 IEEE International Conference on Robotics and Automation, 13-18 May 1990, Los Alamitos, CA, USA, 1990, pp. 1822-7.

[7] K. Liu and F. L. Lewis, "Decentralized continuous robust controller for mobile robots," in Robotics and Automation, 1990. Proceedings., 1990 IEEE International Conference on, 1990, pp. 1822-1827.

[8] J. H. Chung, S. A. Velinsky, and R. A. Hess, "Interaction control of a redundant mobile manipulator," The International Journal of Robotics Research, vol. 17, pp. 1302-1309, 1998.

[9] V. Andaluz, F. Roberti, J. M. Toibero, and R. Carelli, "Adaptive unified motion control of mobile manipulators," Control Engineering Practice, vol. 20, pp. 1337-1352, 2012.

[10] K. Shojaei and A. M. Shahri, "Output feedback tracking control of uncertain non-holonomic wheeled mobile robots: a dynamic surface control approach," Control Theory \& Applications, IET, vol. 6, pp. 216-228, 2012.

[11] Z. Li and S. S. Ge, Fundamentals in Modeling and Control of Mobile Manipulators. vol. 49: CRC Press, 2013.

[12] Y. Kanayama, Y. Kimura, F. Miyazaki, and T. Noguchi, "A stable tracking control method for an autonomous mobile robot," in Robotics and Automation, 1990. Proceedings., 1990 IEEE International Conference on, 1990, pp. 384-389.

[13] M. W. Spong, S. Hutchinson, and M. Vidyasagar, Robot modeling and control. vol. 3: Wiley New York, 2006. 